Original Research Article

\title{
Total thyroidectomy in management of benign thyroid disease
}

\author{
Naik V. ${ }^{1}$, Aithal S.K. ${ }^{2}$ \\ ${ }^{1}$ Dr. Vinay Naik, Senior Resident, Department of General Surgery, Karwar Institute of Medical Sciences, Karwar, \\ Karnataka, ${ }^{2}$ Dr. Srikanth K Aithal, Senior Consultant, Department of General Surgery, St. Martha's Hospital, Bangalore, \\ Karnataka, India.
}

Corresponding Author: Dr. Vinay Naik, Senior Resident, Department of General surgery, Karwar Institute of Medical Sciences, Karwar, Karnataka, India. E-mail: drvinaygnaik@gmail.com

\begin{abstract}
Background: The use of total thyroidectomy in benign thyroid disease treatment is not universal. A total of 60 total thyroidectomies performed for benign thyroid diseases. The Aim of the study was to evaluate the role of total thyroidectomy in patients with presumed bilateral benign thyroid disease, with special emphasis on the incidence of two major post-operative complications namely hypoparathyroidism and recurrent laryngeal nerve palsy. Methods: It was a prospective study, A total of 60 patients who underwent total thyroidectomy for benign thyroid diseases between January 2016 to January 2018 were studied in department of general surgery at St Martha's hospital, Bangalore. The patients were followed up for a period of nine months for post-operative complications. Results: Most of the patients were in the fifth decade of life, Female outnumbered males. Female: male ratio was 4.5:1. Amongst the 60 patients in the present study, the incidence of transient recurrent unilateral laryngeal nerve palsy was seen in $5 \%$, temporary hypocalcemia in $20 \%$, permanent hypocalcemia in $3.3 \%$, and permanent unilateral laryngeal nerve palsy in $1.66 \%$. Malignancy detected after histopathological examination was in $8.33 \%$ of the patients. There was no wound infection, hematoma, bilateral RLN palsy and post-operative mortality. Conclusion: Total thyroidectomy for benign thyroid disease can eliminate any subsequent risk of malignant change in thyroid glands. A low complication rate can be achieved with meticulous surgical technique. Total thyroidectomy can be performed safely and can be considered as valuable option for treating benign bilateral thyroid diseases.
\end{abstract}

Keywords: Benign thyroid disease, Hypoparathyroidism, Recurrent laryngeal nerve palsy.

\section{Introduction}

Total thyroidectomy has an important role in patients with benign thyroid diseases when both lobes of the thyroid gland are involved. The most convenient surgical procedure for benign thyroid diseases is still under controversy. Although the procedure remains controversial total thyroidectomy is increasingly performed. Total thyroidectomy (TT) was suggested as a feasible modality of surgery for benign thyroid disease by Kocher a century ago [1].

Recently, surgery for benign goiter has been recognized as an acceptable and safe treatment modality, which has low surgical risk and mortality rates. Subtotal thyroidectomy that was previously the treatment of choice for benign thyroid disease has been associated with high recurrence rates. It is widely reported that

Manuscript Received: $30^{\text {th }}$ July 2019

Reviewed: $8^{\text {th }}$ August 2019

Author Corrected: $16^{\text {th }}$ August 2019

Accepted for Publication: $21^{\text {st }}$ August 2019 during long-term follow-up after subtotal thyroidectomy in multinodular disease, an increasing recurrence rate of up to $78 \%$ should be expected [2]. A second operation for recurrent nodules is well accepted to result in increased laryngeal nerve and parathyroid morbidity, when compared with an initial approach.

Our aim was to assess whether total thyroidectomy can be carried out safely with low complication rates in presumed benign bilateral thyroid disease in Indian set up.

\section{Materials and Methods}

Study design- This was a Prospective study done to evaluate the safety of total thyroidectomy in bilateral benign thyroid disease with special emphasis on incidence of two major post operative complication namely recurrent laryngeal nerve palsy and hypocalcemia. 


\section{Original Research Article}

Study sample- A total of 60 patients who underwent total thyroidectomy for a condition diagnosed to be benign on preoperative work up including cytology and imaging were prospectively studied from January 2016 to January 2018 at Department of General Surgery, St Martha's Hospital Bangalore. Patient details were collected as per the Proforma. Patients were followed up for minimum period of nine months. Informed and written consent was obtained from all the 60 patients

\section{Inclusion criteria}

- Patients undergoing total thyroidectomy for bilateral benign thyroid disease.

\section{Exclusion criteria}

- Malignancy

- Unilateral involvement

- Surgery for recurrence

Statistical methods: All characteristics were summarized descriptively. For continuous variables the summary statistics of Mean \pm standard deviation were used. For categorical data the number and percentage were used in the data summaries.

Surgical procedure: Approach was through a collar crease incision $2 \mathrm{~cm}$ above suprasternal notch. Subplatysmal flap raised superiorly upto thyroid notch and inferiorly upto suprasternal notch. In midline, strap muscles were split. The dissection of the gland was carried out, middle thyroid vein identified, ligated and divided first, then ligation of the vessels at superior pole of the thyroid gland was carried out.

All the parathyroid glands (At least 3) and Recurrent laryngeal nerve were identified. Then inferior pole dissection was done, capsular branches of the inferior thyroid vessels were ligated to avoid damage to vessels supplying parathyroid gland. If injury to parathyroid gland was suspected or noted, they were re-implanted in the sternocleidomastoid muscle (ipsilateral side).

The gland was then dissected of the pre-tracheal fascia. Similar steps were carried out on contralateral side. And after dissection of the isthmus or pyramidal lobe thyroid gland was delivered. Suction drain was placed in situ, followed by closure of the wound in layers. At the time of extubation, the vocal cord motility were evaluated by anesthesiologist in all patients.

Postoperative management: Postoperatively, a course of antibiotics for $48 \mathrm{hrs}$ was given. Serum calcium was measured on the day of the surgery and on subsequent days in all patients. Calcium level lower than $8.0 \mathrm{mg} / \mathrm{dL}$ (reference range $8.2-10 \mathrm{mg} / \mathrm{dL}$ ) was defined as postoperative hypocalcemia. In asymptomatic patients and patients who did not require any Calcium or Vit D3 supplementation, hypocalcemia resolved within days.

Whereas, symptomatic cases and patients who required calcium supplementation, temporary hypocalcemia was considered to be severe when calcium levels remained lower than $8.0 \mathrm{mg} / \mathrm{dL}$ for more than 3 days.

Hypocalcemia in these patients, resolved within 6 months. Hypoparathyroidism was considered to be permanent in patients who required vitamin $\mathrm{D}$ and calcium supplementation for more than 6 months.

Post operatively, all the patient, after drain removal, were subjected to laryngoscopy for evaluation of vocal cords. Hoarseness of voice associated with vocal cord paralysis on laryngoscopy was defined as recurrent laryngeal nerve palsy. These were considered to be temporary if it resolved within 6 months postoperatively, and permanent if it continued even after 6 months. Hormonal treatment with L-thyroxine began within 5 days after thyroidectomy and after final histopathological diagnosis, in all patients.

Follow-up: A follow-up visits was conducted for all patients. At 1, 2, 3, 6 and 9 months postoperatively, patients were evaluated for parathyroid function, recurrent laryngeal nerve function, hormonal replacement and therapeutic outcome. Routine evaluation included clinical examination, serum thyroid hormones and calcium measurements.

Patients with severe or permanent hypocalcemia were evaluated every 3 months (at 1, 3, 6, 9 months) postoperatively until their serum calcium normalized, with or without vitamin D and calcium supplementation. In patients with recurrent laryngeal nerve palsy, laryngoscopy was done at 1, 6, 9 and 12 months postoperatively to monitor vocal cord function.

\section{Results}

In this prospective study a total of 60 patients who underwent total thyroidectomy for the disease which was diagnosed to be benign on cytology and imaging were analyzed. Patient's demographic details including age, gender and family history according to proforma were noted. 
Original Research Article

Table-1: Age distribution.

\begin{tabular}{|c|c|c|}
\hline Range (in years) & No. of patients & Percentage (\%) \\
\hline $21-30$ & 08 & 13.33 \\
\hline $31-40$ & 12 & 20 \\
\hline $41-50$ & 16 & 26.66 \\
\hline $51-60$ & 11 & 18.33 \\
\hline $61-70$ & 13 & 21.66 \\
\hline
\end{tabular}

Most of the patients were in the fifth decade of their life. None of the patients had previous radiation exposure to his/her neck which increases the risk of thyroid malignancy. The most common symptom at presentation was, swelling in front of neck

Table-2: Sex distribution.

\begin{tabular}{|c|c|c|}
\hline Gender & No of patients & Percentage \\
\hline Male & 11 & 18.33 \\
\hline Female & 49 & 81.66 \\
\hline
\end{tabular}

Female outnumbered males. Female: male ratio was 4.5:1 Amongst all patients, 4 were hyperthyroid at presentation, 36 were euthyroid and the rest i.e. 20 patients were hypothyroid at presentation. All of them were worked up for surgery and had benign diagnosis before surgery.

Table-3: Distribution of cases according to final diagnosis on preoperative work up

\begin{tabular}{|c|c|}
\hline Diagnosis (prior to surgery) & No of patients (\%) \\
\hline Multi Nodular Goitre & $19(31.66)$ \\
\hline Diffuse colloid Goitre & $31(51.66)$ \\
\hline Graves Disease & $02(3.33)$ \\
\hline Thyroiditis & $08(13.33)$ \\
\hline
\end{tabular}

The diagnosis before surgery was multinodular goiter in 19 patients $(31.66 \%)$, diffuse colloid goiter in 31 cases $(51.66 \%)$, Graves disease in 2 cases (3.33\%) and thyroiditis in 8cases (13.33\%).

Table-4: Complications after total thyroidectomy among 60 patients who had surgery

\begin{tabular}{|c|c|c|}
\hline Complication & No of patients & Percentage \\
\hline Hypocalcemia & 12 & $3.33 \%$ \\
\hline Temporary & 2 & \\
\hline Permanent & & \\
\hline Recurrent Laryngeal nerve palsy & & $5 \%$ \\
\hline Temporary & 3 & $0 \%$ \\
\hline Unilateral & 0 & $1.66 \%$ \\
\hline Bilateral & & $0 \%$ \\
\hline Permanent & 1 & $0 \%$ \\
\hline Unilateral & 0 & $0 \%$ \\
\hline Bilateral & 0 & $0 \%$ \\
\hline Haemorrhage & 0 & \\
\hline Wound infection & 0 & \\
\hline Mortality & & \\
\hline
\end{tabular}




\section{Original Research Article}

The most common complication encountered in this study was temporary hypocalcemia which was noted in $12(20 \%)$ patients, temporary hypocalcemia resolved within 6 months. $2(3.33 \%)$ patients had hypocalcemia for 6 months and beyond and it was classified as permanent hypocalcemia. Among the 2 patients with permanent hypocalcemia at 9 months, post operative measurements of serum calcium ranged between $7.6-8.3 \mathrm{mg} / \mathrm{dL}$ with vitamin $\mathrm{D}$ and calcium supplementation. 4 patients who had hoarseness of voice postoperatively were encountered, out of which 3 patients (5\%) had temporary unilateral recurrent laryngeal nerve palsy. All these patients improved within 6-week period postoperatively and only 1 patient $(1.66 \%)$ had permanent recurrent laryngeal nerve weakness. None of the patient had bleeding or hematoma formation after surgery. None of the patient had seroma and wound infection. There was no mortality postoperatively.

Table-5: Histo pathology after total thyroidectomy.

\begin{tabular}{|c|c|}
\hline Thyroid pathology & No (\%) of patients \\
\hline Benign Multi Nodular Goitre & $45(75)$ \\
\hline Graves Disease & $02(3.33)$ \\
\hline Thyroid cancer & $05(8.33)$ \\
\hline Other (Thyroiditis) & $08(13.33)$ \\
\hline
\end{tabular}

In this study group 5 patients had incidental malignancy of thyroid on histopathology examination. These patients were preoperatively diagnosed as nodular colloid goiter on FNAC. Preoperative FNAC was blind procedure not under any image guidance. Most common malignancy was that of papillary carcinoma, All these patients who were diagnosed as malignancy were in the age group ranging from 45 to 65 years and all were females.

\section{Discussion}

The extent of total thyroidectomy in the management of benign thyroid enlargement remains a matter of debate. The only substantial argument for not performing a total thyroidectomy in these patients is the previously reported higher incidence of complications with increasing extents of thyroid resections [3-6]. Total thyroidectomy has an important and increasing role in the management of benign thyroid disease. In some centres, it represents almost half of all thyroid operations that is carried out $[7,8]$. There is increasing recognition that total thyroidectomy is appropriate for patients with multinodular goiter when there is significant nodular disease involving both lobes [9].

Subtotal thyroidectomy was previously been advocated by some for the treatment of bilateral nodular disease, but recurrence rates as high as $45 \%$ have been reported [10-12]. Thyroxine suppressive therapy was once regarded to be valuable method for controlling the progression of multinodular goitre and preventing recurrence after partial thyroidectomy [13].

However, this approach is increasingly being questioned these days, owing to its limited value and potential side-effects specially when used as an adjuvant treatment after subtotal thyroidectomy [10, 11]. Recurrent thyroid disease following sub-total thyroidectomy is a significant problem. Furthermore, the risks involved in reoperation for recurrent multinodular goiter or thyroiditis are significantly higher than after the first operation. The rate of injury to the recurrent laryngeal nerve can be as high as $20 \%$ in patients undergoing reoperation [14]. Beahrs and Vandertoll [6] reported a rate of $9.5 \%$ of permanent recurrent laryngeal nerve palsy and $3.4 \%$ of permanent hypoparathyroidism after a secondary thyroidectomy. Surgical dissection around the RLN during reoperations is difficult because of fibrosis due to previous surgery. The only substantial argument against total thyroidectomy is its potentially higher associated complication rate. However, with appropriate surgical technique, the morbidity of initial total thyroidectomy can be minimized $[15,16]$.

The main aim of surgical treatment for thyroid diseases should always be to perform the most effective treatment with minimum complication and recurrence rates. With introduction of capsular dissection and increasing experience with total thyroidectomies, has made many surgeons to believe that total thyroidectomy is more preferable operation in these cases $[8,14,15$, 17, 18]. Dissection and ligature of the vessels on the thyroid capsule preserve the blood supply of the parathyroid glands and minimize inadvertent injury of the RLN. In our opinion, identification of the RLN during dissection helps prevent accidental injury. Total thyroidectomy can be performed safely in bilateral benign thyroid disease [19]. 


\section{Original Research Article}

In the present study a total of 60 patients who underwent total thyroidectomy for the disease which was diagnosed to be benign on cytology and imaging were analyzed, most of the patients were in the fifth decade of their life. Female outnumbered males. Amongst all the patients, 4 were hyperthyroid, 36 were euthyroid and the rest 20 patients were hypothyroid at presentation. Multinodular goiter in $31.66 \%$ and diffuse colloid goiter in $51.66 \%$ were the two most common benign thyroid diseases identified preoperatively in the present study. In the present study, patients were routinely given L-thyroxine after surgery, only to ensure the restoration of euthyroidism.

The most common complication which was encountered in the present study was that of temporary hypoparathyroidism noted in $20 \%$ of patients. Study conducted by Koyuncu A et al [20] reported temporary hypoparathyroidism in $32.75 \%$ and the study conducted by Gough and Wilkinson et al [7] reported temporary hypoparathyroidism in 30\%. Delbridge et al concluded that a state of transient hypoparathyroidism must be an accepted outcome after bilateral thyroidectomies [8].

The present study showed Temporary recurrent laryngeal nerve palsy in $5 \%$ of our patients. In this study, there were 2 patient who developed permanent hypoparathyroidism and was in follow-up with calcium and vitamin D supplementation. The rate of permanent hypoparathyroidism is $3.33 \%$ in the present study. This compares well with study conducted by Martensson $\mathrm{H}$ et al [21] and Agarwal G [22], which reports similar rates. In study conducted by Koyuncu A et al [20] and Gough and Wilkinson et al [7] rate of permanent hypoparathyroidism was $0 \%$ [22] \& $2.2 \% \quad[14]$ respectively.

Perzik reported an incidence of nerve injury of only $0.4 \%$ [23]. Reeve at al. [17], with their experience with total thyroidectomy observed no permanent complications and had only two cases of temporary cord dysfunction. In the present study it was observed that the permanent RLN palsy in $1.66 \%$ of the patients.

More recently, Liu at al [14] reported a $0.9 \%$ rate of unilateral permanent RLN palsy and no incidence of permanent hypoparathyroidism in 106 consecutive patients who underwent Total thyroidectomy for benign disease. Liu et al [14] confirmed indications of total thyroidectomy, like for multinodular goiter and Graves disease and for patients with inconclusive results on frozen-section analysis and obtained similar low definitive complication rates. In the present study post- operative haemorrhage requiring reoperation did not occur in any patients $(0 \%)$. It was agreed with other authors that hemostasis can be better achieved with total thyroidectomy $[6,24,25]$. There was no wound infection in the present study.

The present study suggests that total thyroidectomy can be carried out with minimum morbidity, among patients with benign thyroid disease. Morbidity is related to the surgeon's experience and technique. Total thyroid gland resections allow the pathologist to study the entire glandular parenchyma and, subsequently, the diagnosis of a higher percentage of malignant lesions.

In the present study group 5 patients $(8.33 \%)$ had incidental malignancy of the thyroid that was picked up on histopathological examination. The most common malignancy was that of papillary carcinoma. Incidental thyroid cancers are detected in $3 \%$ to $16.6 \%$ of apparently benign goiters in numerous studies $(8.33 \%$ in this study) [26-28].

Total thyroidectomy can be used as a treatment modality in multinodular goiter and thyroiditis, when there is bilateral gland involvement, and in Grave's disease because it decreases the likelihood of future repeated operations for recurrent disease [8]. Total thyroidectomy for surgical management of benign thyroid disease can be undertaken safely with a low complication rate.

The main postoperative complications can be avoided by meticulous surgical technique. Total thyroidectomy achieves immediate and permanent cure with no risk of disease recurrence or repeat surgeries

\section{Limitation}

1. Small sample size

2. Short follow up period

3. Single centre trial

\section{Conclusion}

This study has shown that total thyroidectomy can be safely performed with less complication rates in experienced hands and by following meticulous surgical technique. Our data suggests that total thyroidectomy is a valuable option for benign bilateral thyroid disease as it achieves immediate and permanent cure and also can eliminate any subsequent risk of malignant change in thyroid glands with no risk of disease recurrence or redo surgeries. 


\section{Original Research Article}

\section{What this study adds to existing knowledge?}

Total thyroidectomy in benign bilateral thyroid disease can avoid reoperation for recurrence, eliminate any subsequent risk of malignant change. Meticulous surgical technique can lower the complication rates.

\section{Author's contribution}

Dr. Vinay Naik: Study design, Acquisition of data, analysis and interpretation, manuscript submission.

Dr Srikanth K Aithal: Study conception and design, acquisition of data.

Acknowledgment: Author would like to thank St Martha’s hospital, Bangalore.

\section{Abbreviations}

TT: Total Thyroidectomy, RLN: Recurrent Laryngeal Nerve, FNAC: Fine Needle Aspiration Cytology

Conflict of interest: None declared. Funding: Nil, Permission from IRB: Yes

\section{References}

1. Kocher T. Textbook of Operative Surgery, 4th edition, Stiles HJ, translator, London, A. \& C. Black, 1903:535-39.

2. Miccoli P, Antonelli A, Iacconi P, Alberti B, Gambuzza C, Baschieri L. Prospective, randomized, double-blind study about effectiveness of levothyroxine suppressive therapy in prevention of recurrence after operation: result at the third year of follow-up. Surg. 1993; 114(6):1097-1101.

3. Foster RS Jr. Morbidity and mortality after thyroidectomy. Surg Gynecol Obstet. 1978;146(3):423-429.

4. Clark OH. Total thyroidectomy: the treatment of choice for patients with differentiated thyroid cancer. Ann Surg. 1982;196(3):361-370. doi:10.1097/ 00000 658-198209000-00016

5. Harness JK, Fung L, Thompson NW, Burney RE, McLeod MK. Total thyroidectomy: complications and technique. World J. Surg. 1986;10:781. doi:10.1007/ bf01655238.

6. Beahrs $\mathrm{OH}$, Vandertoll DJ. Complications of secondary thyroidectomy. Surg Gynecol Obstet. 1963; 117:535-539.
7. Gough IR, Wilkinson D. Total thyroidectomy for management of thyroid disease. World J Surg. 2000; 24(8):962-965. doi:10.1007/s002680010158

8. Khadra M, Delbridge L, Reeve TS, Poole AG, Crummer P. Total thyroidectomy: its role in the management of thyroid disease. Aust N Z J Surg. 1992; 62 (2):91-95. doi:10.1111/j.1445-2197.1992. tb00003.x

9. Rossi RL, Cady B, Silverman ML, Wool MS, Horner TA. Current results of conservative surgery for differentiated thyroid carcinoma. World J Surg. 1986; 10(4): 612-621. doi:https://doi.org/ 10.1007/ BF01655 538

10. Wadström C, Zedenius J, Guinea A, Reeve T, Delbridge L. Multinodular goitre presenting as a clinical single nodule: how effective is hemithyroidectomy? Aus NZ J Surg. 1999;69(1):34-36. doi: https://doi.org/ 10.1046/j.1440-1622.1999.01485.x

11. Geerdsen JP, Frølund L. Recurrence of nontoxic goitre with and without postoperative thyroxine medication. ClinicEndocrinol.1984;21(5):529-533. doi: https://doi.org/10.1111/j.1365-2265.1984.tb01391.x

12. Röjdmark J, Järhult J. High long term recurrence rate after subtotal thyroidectomy for nodular goitre. Eur J Surg. 1995;161(10):725-727.

13. Berghout A, Wiersinga WM, Touber JL, Smits NJ, Drexhage HA. Comparison of placebo with L-thyroxine alone or with carbimazole for treatment of sporadic non-toxic goitre. The Lancet. 1990;336(8709):193-197. doi: https://doi.org/10.1016/0140-6736(90)91730-X

14. Liu Q, Djuricin G, Prinz RA. Total thyroidectomy for benign thyroid disease. Surg. 1998;123(1): 2-7. doi: https: //doi. org/10.1016/ S0039-6060 (98)70 221-1

15. Bhattacharyya N, Fried MP. Assessment of the morbidity and complications of total thyroidectomy. Arch Otolaryngol Head Neck Surg. 2002;128(4):389392. doi:10.1001/archotol.128.4.389

16. Gardiner KR, Russell CF. Thyroidectomy for large multinodular colloid goitre. J R Coll Surg Edinb. 1995; 40 (6):367-370.

17. Reeve TS, Delbridge L, Cohen A, Crummer P. Total thyroidectomy. The preferred option for multinodular goiter. Ann Surg. 1987;206(6):782-786. 


\section{Original Research Article}

18.O'BrienCJ.Complicationsof thyroidectomy:the benefits of personal audit. Aust NZJ Surg. 1997;67 (1): A25.

19. Sandonato L, Graceffa G, Cipolla C, Fricano S, Acquaro P, Latteri F, Latteri MA. Benign diseases of the thyroid: indications for surgical treatment and the current role of total thyroidectomy. Chirurgia Ital. 2003;55(2):179-187.

20.Koyuncu A, Dökmetas HS, TuranM, Aydin C, Karadayi K, Budak E, et al. Comparison of different thyroidectomy techniques for benign thyroid disease. Endocr J.2003;50(6):723-727.doi:10.1507/endocrj.50.723

21. Mårtensson H, Terins J. Recurrent laryngeal nerve palsy in thyroid gland surgery related to operations and nerves at risk. Arch Surg. 1985;120(4):475-477. doi:10. 1001/archsurg.1985.01390280065014

22. Agarwal G, Aggarwal V. Is total thyroidectomy the surgical procedure of choice for benign multinodular goiter? An evidence-based review. World J Surg. 2008; 32 (7):1313-1324. doi: 10.1007/s00268-008-9579-8.

23. Perzik S. The place of total thyroidectomy in the management of 909 patients with thyroid disease. Am J Surg. 1976;132(4):480-483.doi:10.1016/0002-9610 (76) 90324-x
24. Zambudio AR, Rodríguez J, Riquelme J, Soria T, Canteras M, Parrilla P. Prospective study of postoperative complications after total thyroidectomy for multinodular goiters by surgeons with experience in endocrine surgery. Ann Surg. 2004;240(1):18-25. doi: 10. 1097/01.sla.0000129357.58265.3c

25. Practical management of post-thyroidectomy hematoma. J Surg Oncol. 1994;57(4):235-238. doi:10. 1002/ jso. 2930570406

26. Wheeler MH. Total thyroidectomy for benign thyroid disease. Lancet. 1998; 351(9115):1526-1527. doi: 10.1016/S0140-6736(05)61116-6

27. Delbridge L, Guinea AI, Reeve TS. Total thyroidectomy for bilateral benign multinodular goiter: effect of changing practice. Arch Surg. 1999;134(12): 13891393. doi:10.1001/archsurg.134.12.1389

28. Tezelman S, Borucu I, Senyurek Giles Y, Tunca F, Terzioglu $\mathrm{T}$. The change in surgical practice from subtotal to near-total or total thyroidectomy in the treatment of patients with benign multinodular goiter. World J Surg. 2009;33(3): 400-405. doi:10.1007/ s00268- 008-9808-1.

\section{How to cite this article?}

Naik V, Aithal S.K.Total thyroidectomy in management of benign thyroid disease.Surgical Update: Int $J$ surg Orthopedics. 2019;5(3):227-233.doi:10.17511/ijoso.2019.i03.15 